\title{
Injectivity of the Composition Operators of Étale Mappings
}

\author{
Ronen Peretz \\ Department of Mathematics, Ben Gurion University of the Negev, 84105 Beer Sheva, Israel \\ Correspondence should be addressed to Ronen Peretz; ronenp@math.bgu.ac.il
}

Received 1 May 2014; Revised 10 November 2014; Accepted 19 November 2014; Published 10 December 2014

Academic Editor: Dae San Kim

Copyright (C) 2014 Ronen Peretz. This is an open access article distributed under the Creative Commons Attribution License, which permits unrestricted use, distribution, and reproduction in any medium, provided the original work is properly cited.

\begin{abstract}
Let $X$ be a topological space. The semigroup of all the étale mappings of $X$ (the local homeomorphisms $X \rightarrow X$ ) is denoted by et $(X)$. If $G \in \operatorname{et}(X)$, then the $G$-right (left) composition operator on et $(X)$ is $\operatorname{defined}$ by $R_{G}\left(L_{G}\right)$ : et $(X) \rightarrow \operatorname{et}(X)$, $R_{G}(F)=$ $F \circ G\left(L_{G}(F)=G \circ F\right)$. When are the composition operators injective? The Problem originated in a new approach to study étale polynomial mappings $\mathbb{C}^{2} \rightarrow \mathbb{C}^{2}$ and in particular the two-dimensional Jacobian conjecture. This approach constructs a fractal structure on the semigroup of the (normalized) Keller mappings and outlines a new method of a possible attack on this open problem (in preparation). The construction uses the left composition operator and the injectivity problem is essential. In this paper we will completely solve the injectivity problems of the two composition operators for (normalized) Keller mappings. We will also solve the much easier surjectivity problem of these composition operators.
\end{abstract}

\section{Introduction}

Let $X$ be a topological space. A mapping $F: X \rightarrow X$ is called a local homeomorphism of $X$ or an étale mapping of $X$ if for any point $x \in X$ there exists a neighborhood $U$ of $x$ such that the restriction of $F$ to $U$, denoted by $\left.F\right|_{U}$, is an homeomorphism. The set of all the étale mappings of $X$, denoted by et $(X)$, is a semigroup with a unit with the composition of mappings taken to be the binary operation. If $G \in$ et $(X)$, then the $G$-right composition operator on et $(X)$ is defined by

$$
\begin{gathered}
R_{G}: \text { et }(X) \longrightarrow \operatorname{et}(X) \\
R_{G}(F)=F \circ G .
\end{gathered}
$$

The G-left composition operator on et $(X)$ is defined by

$$
\begin{gathered}
L_{G}: \text { et }(X) \longrightarrow \text { et }(X) \\
L_{G}(F)=G \circ F .
\end{gathered}
$$

We were interested in the injectivity of these two composition operators in two particular cases. The first is the case of entire functions $\mathbb{C} \rightarrow \mathbb{C}$ that are étale (and normalized). The second case is that of the polynomial mappings $\mathbb{C}^{2} \rightarrow \mathbb{C}^{2}$ with determinant of their Jacobian matrix equal (identically) to 1 and whose $Y$-degrees equal their total degrees. For the first case we use the following.

Definition 1. Consider the following

$$
\begin{aligned}
& \operatorname{elh}(\mathbb{C})=\{f: \mathbb{C} \longrightarrow \mathbb{C} \mid f \text { is entire, } \\
&\left.\forall z \in \mathbb{C} f^{\prime}(z) \neq 0, f^{\prime}(0)=1\right\} .
\end{aligned}
$$

Thus we use in this case the symbol elh $(\mathbb{C})$ instead of et $(X)$. Then we have the following.

Proposition 2 (see [1]). Consider the following $\forall f \in \operatorname{elh}(\mathbb{C})$, $R_{f}$ is injective.

Theorem 3 (see [1]). Let $f \in$ elh $(\mathbb{C})$. Then $L_{f}$ is not injective if and only if

$$
f(z)=\frac{1}{b} e^{b z}+a \quad \text { for some } a \in \mathbb{C}, b \in \mathbb{C}^{\times} .
$$

This settled the first case. It should be noted (see [1]) that the proof for the left composition operator is much more involved than the proof for the right composition operator (which follows directly from the Picard little theorem). It is in fact the second case that initiated our interest in the injectivity of the composition operators. It results from a new approach 
to study étale polynomial mappings $\mathbb{C}^{2} \rightarrow \mathbb{C}^{2}$ and in particular the two-dimensional Jacobian conjecture [2-4]. This approach constructs a fractal structure on the semigroup of the (normalized) Keller mappings and outlines a new method of a possible attack on this open problem (in preparation). The construction uses the left composition operator and the injectivity problem is essential. In this paper we will completely solve the injectivity problems of the two composition operators for (normalized) Keller mappings. We will also solve the much easier surjectivity problem of these composition operators.

\section{The Semigroup et $\left(\mathbb{C}^{2}\right)$ of Normalized Keller Mappings and a Few Facts on Their Asymptotic Variety}

Let $F \in \mathbb{C}[X, Y]^{2}$ be an étale mapping that satisfies the two normalizations

(1) $\operatorname{det} J_{F} \equiv 1$;

(2) $\operatorname{deg} P=\operatorname{deg}_{Y} P$ and $\operatorname{deg} Q=\operatorname{deg}_{Y} Q$, where $F(X, Y)=$ $(P(X, Y), Q(X, Y)) \in \mathbb{C}[X, Y]^{2}$.

The set of all such mappings $F$ will be denoted by et $\left(\mathbb{C}^{2}\right)$. This semigroup (with respect to composition of mappings) is the parallel of the semigroup elh $(\mathbb{C})$ for entire functions. The 2dimensional Jacobian conjecture can be rephrased in each of the following forms:

(a) $\operatorname{et}\left(\mathbb{C}^{2}\right) \subseteq \operatorname{Aut}\left(\mathbb{C}^{2}\right)$;

(b) $\left(\mathrm{et}\left(\mathbb{C}^{2}\right)\right.$, o) is a group.

For the next survey of results we refer to the following paper: [5]. We denote by $A(F)$ the asymptotic variety of $F$, that is, the curve of all the asymptotic values of the mapping $F$. The canonical geometric basis of $F$ will be denoted by $R_{0}(F)$. This basis consists of finitely many rational mappings of the following form: $R(X, Y)=\left(X^{-\alpha}, X^{\beta} Y+X^{-\alpha} \Phi(X)\right)$, where $\alpha \epsilon$ $\mathbb{Z}^{+}, \beta \in \mathbb{Z}^{+} \cup\{0\}, \Phi(X) \in \mathbb{C}[X]$, and $\operatorname{deg} \Phi<\alpha+\beta$. Also the effective $X$ powers in $X^{\alpha+\beta} Y+\Phi(X)$ have a gcd which equals 1 . The cardinality of the geometric basis, $\left|R_{0}(F)\right|$, equals the number of components of the affine algebraic curve $A(F)$. $\forall R \in R_{0}(F)$ we have the double asymptotic identity $F$ 。 $R=G_{R} \in \mathbb{C}[X, Y]^{2}$, where the polynomial mapping $G_{R}$ is called the $R$-dual of $F$. Each $R \in R_{0}(F)$ generates exactly one component of $A(F)$. This component is normally parametrized by $\left\{G_{R}(0, Y) \mid Y \in \mathbb{C}\right\}$. We will denote by $H_{R}(X, Y)=0$ the implicit representation of this component in terms of the irreducible polynomial $H_{R} \in \mathbb{C}[X, Y]$. There exists a natural number $\gamma(R) \geq 2$ and a polynomial $S_{R}(X, Y) \in$ $\mathbb{C}[X, Y]$. The affine curve $S_{R}(X, Y)=0$ is called the $R$ phantom curve of $F$. The $R$-component of $A(F), H_{R}(X, Y)=$ 0 , is a polynomial curve, which is not isomorphic to $\mathbb{A}^{1}$, and hence in particular must be a singular irreducible curve. We have the relation $H_{R}\left(G_{R}(X, Y)\right)=X^{\gamma(R)} S_{R}(X, Y)$. The exponent $\gamma(R)$ satisfies the double inequality $2 \leq \gamma(R) \leq \beta-\alpha$.
In our case of the canonical rational mappings $R \in R_{0}(F)$, we have $\sin g(R)=\{X=0\}$. The following is true:

$$
\begin{aligned}
G_{R}^{-1}\left(H_{R}(X, Y)=0\right) & =G_{R}^{-1}\left(G_{R}(\operatorname{sing}(R))\right) \\
& =\operatorname{sing}(R) \cup\left\{S_{R}(X, Y)=0\right\} .
\end{aligned}
$$

Thus the $G_{R}$-preimage of the $R$-component of $A(F)$ (which is the $G_{R}$-image of $\left.\operatorname{sing}(R)\right)$ is the union of two curves: the first is $\operatorname{sing}(R)$ and the second is the so-called $R$-phantom curve of $F$. Even if for a single $R(X, Y)$ the $R$-phantom curve is empty, then $\mathrm{JC}(2)$ follows. Also if $\forall R \in R_{0}(F) \operatorname{sing}(R) \cap\left\{S_{R}(X, Y)=\right.$ $0\}=\emptyset$, then $F$ is a surjective mapping.

Proposition 4. If $F, G \in$ et $\left(\mathbb{C}^{2}\right)$, then $R_{0}(G) \subseteq R_{0}(F \circ G)$, $F(A(G)) \subseteq A(F \circ G)$.

Proof. $R \in R_{0}(G) \Rightarrow G \circ R \in \mathbb{C}[X, Y]^{2} \Rightarrow F \circ(G \circ R) \epsilon$ $\mathbb{C}[X, Y]^{2} \Rightarrow(F \circ G) \circ R \in \mathbb{C}[X, Y]^{2} \Rightarrow R \in R_{0}(F \circ G)$. Next we have $(a, b) \in F(A(G)) \Rightarrow \exists R \in R_{0}(G) \exists Y \in \mathbb{C}$ such that $(a, b)=F((G \circ R)(0, Y)) \Rightarrow \exists R \in R_{0}(F \circ G) \exists Y \in \mathbb{C}$ such that $(a, b)=((F \circ G) \circ R)(0, Y) \Rightarrow(a, b) \in A(F \circ G)$.

The proposition tells us that compositions of étale mappings do not decrease the geometric basis of the right factor and consequently do not decrease the left image of its asymptotic variety. We naturally ask under what conditions the geometric basis of $F \circ G$ is actually larger than that of $G$ ? In other words, we would like to know when is it true that $R_{0}(G) \subset R_{0}(F \circ G)$ ? This happens exactly when $\exists R \in$ $R_{0}(F \circ G)-R_{0}(G)$. This means that $(F \circ G) \circ R \in \mathbb{C}[X, Y]^{2}$, $G \circ R \notin \mathbb{C}[X, Y]^{2}$. Let $R(X, Y)=\left(X^{-\alpha}, X^{\beta} Y+X^{-\alpha} \Phi(X)\right)$, $G(X, Y)=(P(X, Y), Q(X, Y))$. Then

$$
\begin{aligned}
&(G \circ R)(X, Y)=\left(P\left(X^{-\alpha}, X^{\beta} Y+X^{-\alpha} \Phi(X)\right),\right. \\
&\left.Q\left(X^{-\alpha}, X^{\beta} Y+X^{-\alpha} \Phi(X)\right)\right) \\
& \in \mathbb{C}(X, Y)^{2}-\mathbb{C}[X, Y]^{2} .
\end{aligned}
$$

We clearly have $\operatorname{sing}(G \circ R) \subseteq \operatorname{sing}(R)$ and so $\operatorname{sing}(G \circ R)=$ $\{X=0\}$. By $F \circ(G \circ R)=(F \circ G) \circ R \in \mathbb{C}[X, Y]^{2}$ we have $G \circ R \in R(F)$. This is not necessarily a member of the geometric basis of $F$. The canonical geometric basis of $F$, $R_{0}(F)$ contains finitely many rational mappings of the form $S(X, Y)=\left(X^{-a}, X^{b} Y+X^{-a} \Psi(X)\right)$. Since $G \in$ et $\left(\mathbb{C}^{2}\right)$, it follows that $\left|\mathbb{C}^{2}-G\left(\mathbb{C}^{2}\right)\right|<\infty$ (a similar phenomenon as the Picard little theorem). If $L$ is an asymptotic tract of $F$, then $G^{-1}(L)$ cannot be a bounded subset of $\mathbb{C}^{2}$. The reason is that if $\overline{G^{-1}(L)}$ is compact, then $G\left(\overline{G^{-1}(L)}\right)$ is compact and since $L \subseteq$ $G\left(G^{-1}(L)\right) \subseteq G\left(\overline{G^{-1}(L)}\right)$ this would imply the contradiction that $L$ is bounded (and hence cannot be an asymptotic tract). Hence $G^{-1}(L)$ has at least one component, say $L_{1}$, that goes to infinity. This is because the number of components of $G^{-1}(L)$ is finite and $G^{-1}(L)$ is not bounded. So $F \circ G$ has a limit along $L_{1}$ which equals the above asymptotic value of $F$. This proves the following generalization of the second part of Proposition 4, namely, the following proposition. 
Proposition 5. If $F, G \in$ et $\left(\mathbb{C}^{2}\right)$, then $A(F) \cup F(A(G))=$ $A(F \circ G)$.

This proposition implies that if $A(F) \subset F(A(G))$, then necessarily $R_{0}(G) \subset R_{0}(F \circ G)$ because, as shown in the proof of Proposition $4 \forall R \in R_{0}(G),((F \circ G) \circ R)(\operatorname{sing}(R)) \subseteq$ $F(A(G))$.

Proposition 6. Let $F \in$ et $\left(\mathbb{C}^{2}\right)$. If $\exists G \in$ et $\left(\mathbb{C}^{2}\right)$ such that $R_{0}(G)=R_{0}(F \circ G)$, then $F\left(\mathbb{C}^{2}\right)=\mathbb{C}^{2}$; that is, $F$ is a surjective mapping.

Proof. Since $F \in \operatorname{et}\left(\mathbb{C}^{2}\right)$ we have $\mathbb{C}^{2}-F\left(\mathbb{C}^{2}\right) \subseteq A(F)$ because in this case the only points in the complement of the image of $F$ are the finitely many Picard exceptional values of $F$ which are asymptotic values of $F$. If, as the assumption says, $R_{0}(G)=R_{0}(F \circ G)$, then by Proposition 5 we must have $A(F) \subseteq F(A(G)) \subseteq F\left(\mathbb{C}^{2}\right)$ and so there are no Picard exceptional values of the mapping $F$.

If $F \in \operatorname{et}\left(\mathbb{C}^{2}\right)$ is not a surjective mapping, then the last proposition implies that $\forall G \in \operatorname{et}\left(\mathbb{C}^{2}\right)$; we must have $R_{0}(G) \subset$ $R_{0}(F \circ G)$. In particular $R_{0}(F) \subset R_{0}(F \circ F)$. This is the choice $G=F$. If we choose $G=F \circ F$, we get $R_{0}(F \circ F) \subset R_{0}(F \circ(F \circ F))$. Now it is clear that by induction we get the infinite chain of strict inequalities:

$$
\begin{aligned}
R_{0}(F) & \subset R_{0}(F \circ F) \subset R_{0}(F \circ F \circ F) \\
& \subset \cdots \subset R_{0}\left(F^{\circ n}\right) \subset \cdots,
\end{aligned}
$$

where $F^{\circ n}=F \circ \cdots \circ F n$ times fold composition. Since the cardinality of the geometric basis $R_{0}(G)$ is the number of components of the asymptotic variety $A(G)$, it follows in this case that the asymptotic varieties of iterates of $F$ are of increasing complexity in the sense that the number of components of the curve $A\left(F^{o(n+1)}\right)$ is strictly larger than the number of components of the curve $A\left(F^{\text {on }}\right)$. By Proposition 5 we always have $A(F) \cup F(A(F))=A(F \circ F)$. Hence $A(F) \cup$ $F(A(F)) \cup(F \circ F)(A(F))=A(F) \cup F(A(F) \cup F(A(F)))=$ $A(F) \cup F(A(F \circ F))=A(F \circ F \circ F)$. By induction we get in general:

$$
A(F) \cup \bigcup_{k=1}^{n} F^{\circ k}(A(F))=A\left(F^{\circ(n+1)}\right) .
$$

\section{The Composition Operators on et $\left(\mathbb{C}^{2}\right)$ Are Not Surjective, but the Right Composition Operator Is Injective}

Proposition 7. The mappings $R_{F}, L_{F}$ are not surjective if and only if $F \notin$ Aut $\left(\mathbb{C}^{2}\right)$. In fact in this case we have $R_{F}\left(\right.$ et $\left.\left(\mathbb{C}^{2}\right)\right) \subset$ et $\left(\mathbb{C}^{2}\right)-\operatorname{Aut}\left(\mathbb{C}^{2}\right), L_{F}\left(\right.$ et $\left.\left(\mathbb{C}^{2}\right)\right) \subset$ et $\left(\mathbb{C}^{2}\right)-\operatorname{Aut}\left(\mathbb{C}^{2}\right)$.

Proof. Consider the following:

$$
\begin{gathered}
R_{0}\left(R_{F}(G)\right)=R_{0}(G \circ F) \supseteq R_{0}(F) \neq \emptyset, \\
A\left(L_{F}(G)\right)=A(F \circ G) \supseteq A(F) \neq \emptyset .
\end{gathered}
$$

Proposition 8. $R_{F}$ is injective.

Proof. $R_{F}(G)=R_{F}(H) \Rightarrow G \circ F=H \circ F$. Since $F \in$ et $\left(\mathbb{C}^{2}\right)$ we have $\left|\mathbb{C}^{2}-F\left(\mathbb{C}^{2}\right)\right|<\infty$ and by the assumption $\left.G\right|_{F\left(\mathbb{C}^{2}\right)}=$ $\left.H\right|_{F\left(\mathbb{C}^{2}\right)}$. Hence $G \equiv H$.

We naturally inquire if also $L_{F}$ is injective. So let us assume that $L_{F}(G)=L_{F}(H)$. Then $F \circ G=F \circ H$. If we denote $T=F \circ G$, then $R_{0}(G), R_{0}(H) \subseteq R_{0}(T)$ and also $A(F) \cup F(A(G))=A(F) \cup F(A(H))$. If $A(F)=\emptyset$, then $F \in \operatorname{Aut}\left(\mathbb{C}^{2}\right)$ and so $G=F^{-1}(F \circ G)=F^{-1}(F \circ H)=H$. If, on the other hand, $G \neq H$, then there are points $(X, Y) \in \mathbb{C}^{2}$ for which $G(X, Y) \neq H(X, Y)$. By our assumption, $F(G(X, Y))=$ $F(H(X, Y))$, so $F$ is not in $\operatorname{Aut}\left(\mathbb{C}^{2}\right)$ and it identifies different images of $G$ and $H$ of the same $(X, Y)$. We ask the following question: suppose that $F \circ G=F \circ H, G \neq H$, is there a point $(X, Y) \in \mathbb{C}^{2}$ for which $G(X, Y)=H(X, Y)$ ?

Based on our experience with entire functions we tend to prove that the answer to the question is negative. Indeed this is the case and the proof is almost identical to the entire case; see [1]. Namely, if the answer is affirmative, then we have two types of points in $\mathbb{C}^{2}:(U, V) \in \mathbb{C}^{2}$ for which $G(U, V) \neq H(U, V)$ and the complimentary set, where both sets are nonempty. Let us denote by $N$ the first subset of $\mathbb{C}^{2}$; that is,

$$
N=\left\{(U, V) \in \mathbb{C}^{2} \mid G(U, V) \neq H(U, V)\right\} .
$$

The subset $N$ of $\mathbb{C}^{2}$ is open in the strong topology because $G, H$ are étale mappings and if $G(U, V) \neq H(U, V)$, then $\exists O$ an open neighborhood of $(U, V)$ in the strong topology such that $G(O) \cap H(O)=\emptyset$. So the complimentary subset of $N$ is a closed nonempty subset of $\mathbb{C}^{2}$. Let $(X, Y) \in \partial N^{c}$ be a boundary point of $N^{c}$. Let $\left(U_{n}, V_{n}\right) \in N$ satisfy $\lim \left(U_{n}, V_{n}\right)=$ $(X, Y)$. Then $\forall n \in \mathbb{Z}^{+}, G\left(U_{n}, V_{n}\right) \neq H\left(U_{n}, V_{n}\right), F\left(G\left(U_{n}, V_{n}\right)\right)=$ $F\left(H\left(U_{n}, V_{n}\right)\right)$, and $G(X, Y)=H(X, Y)$. This implies that in any strong neighborhood of $G(X, Y)=H(X, Y)$ there are different points, say $G\left(U_{n}, V_{n}\right) \neq H\left(U_{n}, V_{n}\right)$ for $n \in \mathbb{Z}^{+}$large enough, so that $F\left(G\left(U_{n}, V_{n}\right)\right)=F\left(H\left(U_{n}, V_{n}\right)\right)$. Hence $F$ is not injective in any strong neighborhood of $G(X, Y)=H(X, Y)$. Thus $F \notin$ et $\left(\mathbb{C}^{2}\right)$. This contradiction proves the following.

Proposition 9. Suppose that $L_{F}$ is not injective for some $F \in$ et $\left(\mathbb{C}^{2}\right)$. Then $\forall G \neq H, G, H \in$ et $\left(\mathbb{C}^{2}\right)$ such that $L_{F}(G)=$ $L_{F}(H)$ and $\forall(X, Y) \in \mathbb{C}^{2}$ we have $G(X, Y) \neq H(X, Y)$.

Remark 10. Proposition 7 asserts that $\forall F \in$ et $\left(\mathbb{C}^{2}\right)-$ $\operatorname{Aut}\left(\mathbb{C}^{2}\right) \exists G, H \in \operatorname{et}\left(\mathbb{C}^{2}\right)-\operatorname{Aut}\left(\mathbb{C}^{2}\right)$ such that $\forall M \in \operatorname{et}\left(\mathbb{C}^{2}\right)$ we have $M \circ F \neq G$ and $F \circ M \neq H$.

\section{The Size, $d_{F}$, of the Generic Fiber of a Keller Mapping $F \in \operatorname{et}\left(\mathbb{C}^{2}\right)$}

We will need the generic size of a fiber of a mapping $F=$ $(P, Q) \in$ et $\left(\mathbb{C}^{2}\right)$. If we denote $\operatorname{deg} P(X, Y)=n$ and $\operatorname{deg} Q(X, Y)=m$, then $\forall(a, b) \in \mathbb{C}^{2}$ the $F$ fiber over $(a, b)$ is $F^{-1}(a, b)=\left\{(x, y) \in \mathbb{C}^{2} \mid F(x, y)=F(a, b)\right\}$. It is well known that this set is a finite subset of $\mathbb{C}^{2}$ and, by the Bezout theorem, 
we have $\left|\left\{(x, y) \in \mathbb{C}^{2} \mid F(x, y)=(a, b)\right\}\right|=\left|F^{-1}(a, b)\right| \leq n \cdot m$. Moreover, there is a number that we will denote by $d_{F}$ such that generically in $(a, b)$ we have $\left|F^{-1}(a, b)\right|=d_{F}$. This means that $\left\{(a, b) \in \mathbb{C}^{2}|| F^{-1}(a, b) \mid \neq d_{F}\right\}$ is a closed and proper Zariski subset of $\mathbb{C}^{2}$. In fact $\forall(a, b) \in \mathbb{C}^{2},\left|F^{-1}(a, b)\right| \neq d_{F} \Rightarrow$ $\left|F^{-1}(a, b)\right|<d_{F}$. Thus we have $d_{F}=\max \left\{\left|F^{-1}(a, b)\right| \mid(a, b) \in\right.$ $\left.\mathbb{C}^{2}\right\}$.

Definition 11. Let $F \in \operatorname{et}\left(\mathbb{C}^{2}\right)$. We will denote $d_{F}=$ $\max \left\{\left|F^{-1}(a, b)\right| \mid(a, b) \in \mathbb{C}^{2}\right\}$. We will call $d_{F}$ the geometrical degree of the étale mapping $F$.

Proposition 12. Consider the following $\forall F, G \in$ et $\left(\mathbb{C}^{2}\right)$, $d_{F \circ G}=d_{F} \cdot d_{G}$.

This is a well-known result. We include one of its proofs for convenience.

Proof. $\forall(a, b) \in \mathbb{C}^{2},(F \circ G)^{-1}(a, b)=G^{-1}\left(F^{-1}(a, b)\right)$. But generically in $(a, b),\left|F^{-1}(a, b)\right|=d_{F}$ and generically in $(c, d)$, $\left|G^{-1}(c, d)\right|=d_{G}$.

Definition 13. An étale mapping $F \in$ et $\left(\mathbb{C}^{2}\right)$ is composite if $\exists G, H \in \operatorname{et}\left(\mathbb{C}^{2}\right)-\operatorname{Aut}\left(\mathbb{C}^{2}\right)$ such that $F=G \circ H$. An étale mapping $A \in \operatorname{et}\left(\mathbb{C}^{2}\right)-\operatorname{Aut}\left(\mathbb{C}^{2}\right)$ is prime if it is not composite. This is equivalent to $A=B \circ C$ for some $B, C \in \operatorname{et}\left(\mathbb{C}^{2}\right) \Rightarrow B \in$ $\operatorname{Aut}\left(\mathbb{C}^{2}\right) \vee C \in \operatorname{Aut}\left(\mathbb{C}^{2}\right)$. The subset of et $\left(\mathbb{C}^{2}\right)$ of all the prime mappings will be denoted by et ${ }_{p}\left(\mathbb{C}^{2}\right)$. Thus the set of all the composite étale mappings is et $\left(\mathbb{C}^{2}\right)-\mathrm{et}_{p}\left(\mathbb{C}^{2}\right)$.

Proposition 14. $\forall F \in$ et $\left(\mathbb{C}^{2}\right)-$ et $_{p}\left(\mathbb{C}^{2}\right), d_{F}$ is not a prime number. Equivalently, $\forall F \in$ et $\left(\mathbb{C}^{2}\right), d_{F}$ is a prime number $\Rightarrow F \in \mathrm{et}_{p}\left(\mathbb{C}^{2}\right)$.

Proof. $F \in \operatorname{et}\left(\mathbb{C}^{2}\right)-\mathrm{et}_{p}\left(\mathbb{C}^{2}\right) \Rightarrow \exists G, H \in \operatorname{et}\left(\mathbb{C}^{2}\right)-\operatorname{Aut}\left(\mathbb{C}^{2}\right)$ such that $F=G \circ H$ (by the definition) $\Rightarrow d_{F}=d_{G} \cdot d_{H}, d_{G}, d_{H}>$ 1 (by Proposition 12 and the fact that $d_{M}=1 \Leftrightarrow M \in$ $\left.\operatorname{Aut}\left(\mathbb{C}^{2}\right)\right) \Rightarrow d_{F}$ is a composite integer.

Theorem 15. The following hold true.

(1) If et $\left(\mathbb{C}^{2}\right)-$ Aut $\left(\mathbb{C}^{2}\right) \neq \emptyset$, then et ${ }_{p}\left(\mathbb{C}^{2}\right) \neq \emptyset$.

(2) $\forall F \in$ et $\left(\mathbb{C}^{2}\right) \exists k \in \mathbb{Z}^{+} \cup\{0\} \quad \exists A_{0} \in \operatorname{Aut}\left(\mathbb{C}^{2}\right)$ $\exists P_{1}, \ldots, P_{k} \in$ et $_{p}\left(\mathbb{C}^{2}\right)$ such that $F=A_{0} \circ P_{1} \circ \cdots \circ P_{k}$.

Proof. If et ${ }_{p}\left(\mathbb{C}^{2}\right)=\emptyset$, then et $\left(\mathbb{C}^{2}\right)-\operatorname{Aut}\left(\mathbb{C}^{2}\right)$ are all composite étale mappings. Let $F \in \operatorname{et}\left(\mathbb{C}^{2}\right)-\operatorname{Aut}\left(\mathbb{C}^{2}\right)$, then $\exists G_{1}, G_{2}^{\prime} \in$ et $\left(\mathbb{C}^{2}\right)-\operatorname{Aut}\left(\mathbb{C}^{2}\right)$ such that $F=G_{1} \circ G_{2}^{\prime}$. So $\exists G_{2}, G_{3}^{\prime} \in \operatorname{et}\left(\mathbb{C}^{2}\right)-$ $\operatorname{Aut}\left(\mathbb{C}^{2}\right)$ such that $G_{2}^{\prime}=G_{2} \circ G_{3}^{\prime}$. Hence $F=G_{1} \circ G_{2} \circ G_{3}^{\prime}$. Continuing this we get for any $k \in \mathbb{Z}^{+} \exists G_{1}, \ldots, G_{k} \in \operatorname{et}\left(\mathbb{C}^{2}\right)-$ $\operatorname{Aut}\left(\mathbb{C}^{2}\right)$ such that $F=G_{1} \circ \cdots \circ G_{k}$ and by Proposition $12 d_{F}=$ $\prod_{j=1}^{k} d_{G_{j}}$. But $\forall 1 \leq j \leq k, d_{G_{j}} \geq 2$ and so $\forall k \in \mathbb{Z}^{+}, d_{F} \geq 2^{k}$, a contradiction to $d_{F}<\infty$. Thus et ${ }_{p}\left(\mathbb{C}^{2}\right) \neq \emptyset$.

Now part 2 is standard, for if $F \in \operatorname{Aut}\left(\mathbb{C}^{2}\right)$ we take $A_{0}=F$ and $k=0$. If $F \in$ et $_{p}\left(\mathbb{C}^{2}\right)$ we take $A_{0}=\mathrm{id}, k=1$, and $P_{1}=F$. If $F \in \operatorname{et}\left(\mathbb{C}^{2}\right)-$ et $_{p}\left(\mathbb{C}^{2}\right)$, then $F=G \circ H$ for some $G, H \in$ et $\left(\mathbb{C}^{2}\right)-\operatorname{Aut}\left(\mathbb{C}^{2}\right)$. So by Proposition $12 d_{F}=d_{G} \cdot d_{H}$ and since $d_{G}, d_{H} \geq 2$ it follows that $d_{G}, d_{H}<d_{F}$ and we conclude the proof of part 2 using induction on the geometrical degree. Namely, $G=P_{1} \circ \cdots \circ P_{m}, H=P_{m+1} \circ \cdots \circ P_{k}$ for $m \geq 1$, $k \geq m+1$, and some primes $P_{1}, \ldots, P_{k} \in$ et $_{p}\left(\mathbb{C}^{2}\right)$.

\section{The Metric Spaces $\left(\mathrm{et}\left(\mathbb{C}^{2}\right), \rho_{D}\right)$}

We will need a special kind of four (real) dimensional subsets of $\mathbb{R}^{4}$. These will serve us to construct suitable metric structures on et $\left(\mathbb{C}^{2}\right)$.

Definition 16. Let $D$ be an open subset of $\mathbb{C}^{2}$ with respect to the strong topology that satisfies the following conditions.

(1) $\operatorname{int}(\bar{D})=D(D$ has no "slits").

(2) $\bar{D}$ is a compact subset of $\mathbb{C}^{2}$ (in the strong topology).

(3) $\forall G_{1}, G_{2} \in \operatorname{et}\left(\mathbb{C}^{2}\right), G_{1}(D)=G_{2}(D) \Leftrightarrow G_{1}=G_{2}$.

We define the following real valued function:

$$
\begin{gathered}
\rho_{D}: \text { et }\left(\mathbb{C}^{2}\right) \times \text { et }\left(\mathbb{C}^{2}\right) \longrightarrow \mathbb{R}^{+} \cup\{0\}, \\
\rho_{D}\left(G_{1}, G_{2}\right)=\text { the volume of } G_{1}(D) \Delta G_{2}(D) .
\end{gathered}
$$

Here we use the standard set-theoretic notation of the symmetric difference between two sets $A$ and $B$; that is, $A \Delta B=(A-B) \cup(B-A)$.

Remark 17. It is not clear how to construct an open subset $D$ of $\mathbb{C}^{2}$ that will satisfy the three properties that are required in Definition 16. We will postpone for a while the demonstration that such open sets exist.

Proposition 18. $\rho_{D}$ is a metric on et $\left(\mathbb{C}^{2}\right)$.

Proof. (1) $\rho_{D}\left(G_{1}, G_{2}\right)=0 \Leftrightarrow$ the volume of $G_{1}(D) \Delta G_{2}(D)=$ $0 \Leftrightarrow G_{1}(D)=G_{2}(D)$ (where the last equivalence follows by the fact that $G_{1}$ and $G_{2}$ are local homeomorphisms in the strong topology and because of condition (1) in Definition 16) $\Leftrightarrow G_{1}=G_{2}$ (by condition (3) in Definition 16).

(2) By $G_{1}(D) \Delta G_{2}(D)=G_{2}(D) \Delta G_{1}(D)$, it follows that $\rho_{D}\left(G_{1}, G_{2}\right)=\rho_{D}\left(G_{2}, G_{1}\right)$.

(3) Here we use a little technical set-theoretic containment. Namely, for any three sets $A, B$, and $C$ we have

$$
A \Delta C \subseteq(A \Delta B) \cup(B \Delta C)
$$

This implies that $G_{1}(D) \Delta G_{3}(D) \subseteq\left(G_{1}(D) \Delta G_{2}(D)\right) \cup\left(G_{2}(D) \Delta\right.$ $\left.G_{3}(D)\right)$ from which it follows that

$$
\begin{aligned}
& \text { (the volume of } \left.G_{1}(D) \Delta G_{3}(D)\right) \\
& \leq\left(\text { the volume of } G_{1}(D) \Delta G_{2}(D)\right) \\
& \quad+\left(\text { the volume of } G_{2}(D) \Delta G_{3}(D)\right) .
\end{aligned}
$$

Hence the triangle inequality $\rho_{D}\left(G_{1}, G_{3}\right) \leq \rho_{D}\left(G_{1}, G_{2}\right)+$ $\rho_{D}\left(G_{2}, G_{3}\right)$ holds. 
So far we thought of the volume of $G_{1}(D) \Delta G_{2}(D)$ as the volume of the open set which is the symmetric difference between the $G_{1}$ image and the $G_{2}$ image of the open set $D$. However, the mappings $G_{1}$ and $G_{2}$ are étale and in particular need not be injective. We will take into the volume computation the multiplicities of $G_{1}$ and of $G_{2}$. By Theorem 3 on page 39 of [6] we have the following: given that $F: \mathbb{C}^{n} \rightarrow$ $\mathbb{C}^{n}$ we define $\widetilde{F}=\left(\operatorname{Re} F_{1}, \operatorname{Im} F_{1}, \ldots, \operatorname{Re} F_{n}, \operatorname{Im} F_{n}\right) ; \mathbb{R}^{2 n} \rightarrow$ $\mathbb{R}^{2 n}$. Then $\operatorname{det} J_{\widetilde{F}}=\left|\operatorname{det} J_{F}\right|^{2}$. Thus the Jacobian condition $\operatorname{det} J_{F} \equiv 1$ implies that $\operatorname{det} J_{\widetilde{F}} \equiv 1$. So the real mapping $\widetilde{F}$ preserves the usual volume form. In order to take into account the multiplicities of the étale mappings $G_{1}$ and $G_{2}$ when computing the volume of the symmetric difference $G_{1}(D) \Delta G_{2}(D)$ we had to do the following. For any $G \in \operatorname{et}\left(\mathbb{C}^{2}\right)$ instead of computing,

$$
\iiint \int_{D}\left(\operatorname{det} J_{\widetilde{G}} \cdot d V\right)=\iiint \int_{D} d V
$$

we compute

$$
\iiint \int_{\widetilde{\mathrm{G}}(D)} d X_{1} d X_{2} d Y_{1} d Y_{2}
$$

$$
\text { where } X=X_{1}+i X_{2}, \quad Y=Y_{1}+i Y_{2} \text {. }
$$

For every $j=1,2, \ldots, d_{G}$ we denote by $D_{j}$ the subset of $D$ such that for each point of $D_{j}$ there are exactly $j$ points of $D$ that are mapped by $G$ to the same image of that point. In other words, $D_{j}=\left\{\alpha \in D|| \widetilde{G}^{-1}(\widetilde{G}(\alpha)) \cap D \mid=j\right\}$. We assume that $D$ is large enough so that $\forall j=1, \ldots, d_{G}$ we have $D_{j} \neq \emptyset$. For our étale mappings it is well known that if $j<d_{G}$, then $\operatorname{dim} D_{j}<\operatorname{dim} D$, so the volume of these $D_{j}$ 's contribution equals 0 . The dimension claim follows by the well-known fact that the size of a generic fiber $\left|G^{-1}(x)\right|$ equals $d_{G}$ and that $d_{G}$ is also the maximal size of any of the fibers of $G$. However, for the sake of treating more general families of mappings, we denote by $\operatorname{vol}\left(D_{j}\right)$ the volume of the set $D_{j}$. Then $D$ has a partition into exactly $j$ subsets of equal volume. The volume of each such a set is $\operatorname{vol}\left(D_{j}\right) / j$ and each such a set has exactly one of the $j$ points in $\widetilde{G}^{-1}(\widetilde{G}(\alpha)) \cap D$ for each $\alpha \in D_{j}$. We note that $\operatorname{vol}\left(\widetilde{G}\left(D_{j}\right)\right)=\operatorname{vol}\left(D_{j}\right) / j$ by the Jacobian Condition. Thus the volume with the multiplicity of $\widetilde{G}$ taken into account is given by

$$
\begin{aligned}
& \operatorname{vol}(\widetilde{G}(D))+\sum_{j=2}^{d_{G}}(j-1) \cdot \frac{\operatorname{vol}\left(D_{j}\right)}{j} \\
& =\operatorname{vol}(\widetilde{G}(D))+\sum_{j=2}^{d_{G}}(j-1) \cdot \operatorname{vol}\left(\widetilde{G}\left(D_{j}\right)\right) .
\end{aligned}
$$

We note that $\widetilde{G}(D)=\bigcup_{j=1}^{d_{G}} \widetilde{G}\left(D_{j}\right)$ is a partition, so $\operatorname{vol}(\widetilde{G}(D))=\sum_{j=1}^{d_{G}} \operatorname{vol}\left(\widetilde{G}\left(D_{j}\right)\right)$. Hence we can express the desired volume by

$$
\begin{aligned}
& \operatorname{vol}(\widetilde{G}(D))+\sum_{j=2}^{d_{G}}(j-1) \cdot \operatorname{vol}\left(\widetilde{G}\left(D_{j}\right)\right) \\
& =\sum_{j=1}^{d_{G}} j \cdot \operatorname{vol}\left(\widetilde{G}\left(D_{j}\right)\right) .
\end{aligned}
$$

We note that this equals $\sum_{j=1}^{d_{G}} \operatorname{vol}\left(D_{j}\right)$ and since $D=\bigcup_{j=1}^{d_{G}} D_{j}$ is a partition we have $\operatorname{vol}(D)=\sum_{j=1}^{d_{G}} \operatorname{vol}\left(D_{j}\right)$. As expected, the volume computation that takes into account the multiplicity of $G$ is in general larger than the geometric volume $\operatorname{vol}(\widetilde{G}(D))$. The access can be expressed in several forms:

$$
\begin{aligned}
\operatorname{vol}(D)-\operatorname{vol}(\widetilde{G}(D)) & =\sum_{j=2}^{d_{G}}(j-1) \cdot \operatorname{vol}\left(\widetilde{G}\left(D_{j}\right)\right) \\
& =\sum_{j=2}^{d_{G}}\left(1-\frac{1}{j}\right) \operatorname{vol}\left(D_{j}\right) .
\end{aligned}
$$

Coming back to the computation of the metric distance $\rho_{D}\left(G_{1}, G_{2}\right)=$ the volume of $G_{1}(D) \Delta G_{2}(D)$ we compute the volume of $G_{1}(D)-G_{2}(D)$ with the multiplicity of $G_{1}$ while the volume of $G_{2}(D)-G_{1}(D)$ is computed with the multiplicity of $G_{2}$.

\section{Characteristic Sets of Families of Holomorphic Local Homeomorphisms $\mathbb{C}^{2} \rightarrow \mathbb{C}^{2}$}

In this section we prove the existence of sets $D$ that satisfy the three properties that are required in Definition 16. The third property will turn out to be the tricky one.

Definition 19. Let $\Gamma$ be a family of holomorphic local homeomorphisms $F: \mathbb{C}^{2} \rightarrow \mathbb{C}^{2}$. A subset $D \subseteq \mathbb{C}^{2}$ is called a characteristic set of $\Gamma$ if it satisfies the following condition: $\forall F_{1}, F_{2} \in \Gamma, F_{1}(D)=F_{2}(D) \Leftrightarrow F_{1}=F_{2}$.

We start by recalling the well-known rigidity property of holomorphic functions in one complex variable. Also known as the permanence principle, or the identity theorem. The identity theorem for analytic functions of one complex variable says that if $D \subseteq \mathbb{C}$ is a domain (an open and a connected set) and if $E$ is a subset of $D$ that has a nonisolated point and if $f(z)$ is an analytic function defined on $D$ and vanishing on $E$, then $f(z)=0$ for all $z \in D$.

There is an identity theorem for analytic functions in several complex variables, but for more than one variable the above statement is false. One correct statement is as follows: "Let $f(z)$ and $g(z)$ be holomorphic functions in a domain $D$ in $\mathbb{C}^{n}$. If $f(z)=g(z)$ for all $z$ in a nonempty set $\delta$ in $D$, then $f(z)=g(z)$ in $D$. Hence, analytic continuation of holomorphic functions in several complex variables can be performed as in the case of one complex variable. Contrary to the case of one complex variable, the zero set of a holomorphic function in a domain $D \subseteq \mathbb{C}^{n}, n \geq$ 2 , contains no isolated points. Thus even if $f(z)=g(z)$ in a set 
with accumulation points in $D$, it does not necessarily follow that $f(z)=g(z)$ in $D$. For example, in $\mathbb{C}^{2}$ with variables $z$ and $w$ we can take $f(z, w)=z$ and $g(z, w)=z^{2 ”}$ [7, Chapter 1, page 16].

In spite of the above standard identity theorem for $n \geq 2$ complex variables that requires a thick set $E$ (i.e., an open set) on which $f(z)=g(z)$ one can do much better. Let us start with the following. Let $F(Z, W)$ be an entire function of two complex variables $Z$ and $W$. Let us define a subset $E$ of $\mathbb{C}^{2}$ as follows. We take a convergent sequence $\left\{Z_{k}\right\}_{k=1}^{\infty}$ of different numbers. Thus $\lim Z_{k}=a$ and $j \neq k \Rightarrow Z_{j} \neq Z_{k}$. For each $k$, let $\left\{W_{j}^{(k)}\right\}_{j=1}^{\infty}$ be a convergent sequence of different numbers, such that their $\operatorname{limit}_{\text {im }} \lim _{j \rightarrow \infty} W_{j}^{(k)}=Z_{k}^{\prime}$. We define $E=$ $\left\{\left(Z_{k}, W_{j}^{(k)}\right) \mid j, k=1,2,3, \ldots\right\}$. Now we have the following.

Proposition 20. If $F(Z, W)$ vanishes on $E$, that is, $F\left(Z_{k}, W_{j}^{(k)}\right)=0$ for $j, k=1,2,3, \ldots$, then $F(Z, W) \equiv 0$ is the zero function.

Remark 21. We note that $E$ is a thin set, in fact a countable set. Even the closure $\bar{E}$ is thin.

Proof of Proposition 20. Since $F(Z, W)$ is an entire function, it can be represented as a convergent power series centered at $(0,0)$ with an infinite radius of convergence. We can sum the terms in the order we please. Let us write $F(Z, W)$ as a power series in $W$ with coefficients that are entire functions in $Z$. Thus we have $F(Z, W)=\sum_{k=0}^{\infty} a_{k}(Z) W^{k}$, where for each $k=0,1,2, \ldots, a_{k}(Z)$ is an entire function in the variable $Z$. For a fixed $l \in\{1,2,3, \ldots\}$ we have by our assumptions the following: $F\left(Z_{l}, W_{j}^{(l)}\right)=0$ for $j=1,2,3, \ldots$. But $\lim _{j \rightarrow \infty} W_{j}^{(l)}=Z_{l}^{\prime}$ so that $g_{l}(W)=F\left(Z_{l}, W\right)$ is an entire function of the single variable $W$, which vanishes on a convergent sequence $\left\{W_{j}^{(l)}\right\}_{j=1}^{\infty}$. By the identity theorem of one complex variable we deduce that $g_{l}(W) \equiv 0$, the zero function. Since $g_{l}(W)=\sum_{k=0}^{\infty} a_{k}\left(Z_{l}\right) W^{k}$ it follows that the Maclaurin coefficients $a_{k}\left(Z_{l}\right), k=0,1,2, \ldots$ vanish. Now, this is valid for each $l$, and $\lim Z_{l}=a$ converges. Since each $a_{k}(Z)$ is an entire function which vanishes on a convergent sequence $\left\{Z_{l}\right\}_{l=1}^{\infty}$ it follows, once again, by the identity theorem in one complex variable, that is, $a_{k}(Z) \equiv 0$, the zero function $k=0,1,2, \ldots$. Hence we conclude that $F(Z, W)=\sum_{k=0}^{\infty} a_{k}(Z) W^{k} \equiv 0$.

This type of elementary arguments that was used to construct a thin set $E$ for identity purpose is not new. For example, see the following.

Theorem 22 (see [8]). Let $D \subseteq \mathbb{C}$ be a domain, and let $E$ be a subset of $D$ that has a nonisolated point. Let $F(Z, W)$ be a function defined for $Z, W \in D$ such that $F(Z, W)$ is analytic in $Z$ for each fixed $W \in D$ and analytic in $W$ for each fixed $Z \in D$. If $F(Z, W)=0$ whenever $Z$ and $W$ both belong to $E$, then $F(Z, W)=0$ for all $Z, W \in D$.

Advancing along the lines of the construction of the thin set in Proposition 20 we note that if $\left\{Z_{k}\right\}_{k=1}^{\infty}$ is a sequence of different numbers that converges to $\lim Z_{k}=a$, and if for each $k=1,2,3, \ldots$ there is a straight line segment $\left[\alpha_{k}, \beta_{k}\right]$ of $W$ 's such that two entire functions $F(Z, W)$ and $G(Z, W)$ agree on the union (a countable union) of the segments $\left\{Z_{k}\right\} \times$ $\left[\alpha_{k}, \beta_{k}\right]$, that is, $F\left(Z_{k}, W\right)=G\left(Z_{k}, W\right), \forall W \in\left[\alpha_{k}, \beta_{k}\right]$, then $F(Z, W) \equiv G(Z, W), \forall(Z, W) \in \mathbb{C}^{2}$.

We now will construct characteristic sets of families $\Gamma$ of holomorphic local homeomorphisms $F: \mathbb{C}^{2} \rightarrow \mathbb{C}^{2}$.

Definition 23. Let $m$ be a natural number and $\alpha \in \mathbb{C}^{2}$. An $m$-star at $\alpha$ is the union of $m$ line segments, so that any pair intersects in $\alpha$.

Definition 24. Let $l$ be a line segment and let $\left\{\alpha_{k}\right\}$ be a countable dense subset of $l$. Let $\left\{n_{k}\right\}$ be a sequence of different natural numbers and $\forall k$, let $S_{n_{k}}$ be an $n_{k}$-star at $\alpha_{k}$ such that one of the star's segments lies on $l$, and such that $\forall k_{1} \neq k_{2}$, $\widetilde{S}_{n_{k_{1}}} \cap \widetilde{S}_{n_{k_{2}}}=\emptyset$. Here we denoted $\widetilde{S}=S-l$. Moreover, we group the stars in bundles of, say 5 , thus getting the sequence of star bundles:

$$
\begin{aligned}
& \left\{S_{n_{1}}, S_{n_{2}}, S_{n_{3}}, S_{n_{4}}, S_{n_{5}}\right\}, \\
& \left\{S_{n_{6}}, \ldots, S_{n_{10}}\right\}, \ldots,\left\{S_{n_{5 j+1}}, \ldots, S_{n_{5 j+5}}\right\}, \ldots
\end{aligned}
$$

and for each bundle of five we take the maximal length of its rays to be at most $1 / 10$ the length of the maximal length of the previous bundle. We define

$$
l_{0}^{\left\{n_{k}\right\}}=l \cup \bigcup_{k=1}^{\infty} S_{n_{k}} .
$$

Let $\left\{Z_{k}\right\}_{k=1}^{\infty}$ be a sequence of different complex numbers that converges to $\lim Z_{k}=a$. Let $\left\{\left\{n_{j}^{(k)}\right\}_{j=1}^{\infty}\right\}_{k=1}^{\infty}$ be a partition of the natural numbers, $\mathbb{Z}^{+}$. In fact all we need is the disjointness, that is, $k_{1} \neq k_{2} \Rightarrow\left\{n_{j}^{\left(k_{1}\right)}\right\}_{j=1}^{\infty} \cap\left\{n_{j}^{\left(k_{2}\right)}\right\}_{j=1}^{\infty}=\emptyset$. Let us consider the starred segments

$$
\left\{l_{0}^{\left\{n_{j}^{(k)}\right\}_{j=1}^{\infty}} \mid k=1,2,3, \ldots\right\}
$$

and define the following countable union of starred segments in $\mathbb{C}^{2}$ :

$$
\bigcup_{k=1}^{\infty}\left\{Z_{k}\right\} \times l_{0}^{\left\{n_{j}^{(k)}\right\}_{j=1}^{\infty}},
$$

where we assume that the lengths of the star rays were chosen to satisfy disjointness in $\mathbb{C}^{2}$; namely,

$$
k_{1} \neq k_{2} \Longrightarrow\left\{Z_{k_{1}}\right\} \times l_{0}^{\left\{n_{j}^{\left(k_{1}\right)}\right\}_{j=1}^{\infty}} \cap\left\{Z_{k_{2}}\right\} \times l_{0}^{\left\{n_{j}^{\left(k_{2}\right)}\right\}_{j=1}^{\infty}}=\emptyset .
$$

We let

$$
E=\bigcup_{k=1}^{\infty}\left\{Z_{k}\right\} \times l_{0}^{\left\{n_{j}^{(k)}\right\}_{j=1}^{\infty}},
$$

or if we need a closed (compact) set, the closure of this union. 
Proposition 25. Let $\Gamma$ be any family of entire holomorphic local homeomorphisms $F: \mathbb{C}^{2} \rightarrow \mathbb{C}^{2}$. Then $E$ is a characteristic set of $\Gamma$.

Proof. Let $F_{1}, F_{2} \in \Gamma$ satisfy $F_{1}(E)=F_{2}(E)$. Then each starred line segment

$$
\left\{Z_{k}\right\} \times l_{0}^{\left\{n_{j}^{(k)}\right\}_{j=1}^{\infty}}
$$

must be mapped onto a curve

$$
F_{1}\left(\left\{Z_{k}\right\} \times l_{0}^{\left\{n_{j}^{(k)}\right\}_{j=1}^{\infty}}\right)=F_{2}\left(\left\{Z_{k}\right\} \times l_{0}^{\left\{n_{j}^{(k)}\right\}_{j=1}^{\infty}}\right)
$$

and each $n_{j}^{(k)}$-star on $l, S_{n_{j}^{(k)}}$ is mapped onto a holomorphic $n_{j}^{(k)}$-star

$$
F_{1}\left(\left\{Z_{k}\right\} \times S_{n_{j}^{(k)}}\right)=F_{2}\left(\left\{Z_{k}\right\} \times S_{n_{j}^{(k)}}\right) .
$$

This is because the valence sequences of the stars

$$
\left\{\left\{n_{j}^{(k)}\right\}_{j=1}^{\infty}\right\}_{k=1}^{\infty}
$$

are pairwise disjoint natural numbers, and $F_{1}, F_{2}$ are local homeomorphisms and hence preserve the star valencies $n_{j}^{(k)}$. The centers of the holomorphic stars

$$
\left\{F_{1}\left(\alpha_{n_{j}^{(k)}}\right)\right\}=\left\{F_{2}\left(\alpha_{n_{j}^{(k)}}\right)\right\}
$$

form a countable and a dense subset of the curves $F_{1}\left(\left\{Z_{k}\right\} \times\right.$ $l)=F_{2}\left(\left\{Z_{k}\right\} \times l\right)$. By continuity this implies that the restrictions

$$
\left.F_{1}\right|_{\left\{Z_{k}\right\} \times l},\left.\quad F_{2}\right|_{\left\{Z_{k}\right\} \times l}
$$

coincide. Since $F_{1}$ and $F_{2}$ are holomorphic, this implies by Proposition 20 (which is a variant of the identity theorem for entire functions $\mathbb{C}^{2} \rightarrow \mathbb{C}^{2}$ ) that $F_{1} \equiv F_{2}$.

Remark 26. Proposition 25 holds true for any rigid family of local homeomorphisms. Rigidity here means that

$$
\left.F_{1}\right|_{\left\{Z_{k}\right\} \times l}=\left.F_{2}\right|_{\left\{Z_{k}\right\} \times l} \Longleftrightarrow F_{1} \equiv F_{2} .
$$

So the proposition holds true for holomorphic mappings, for harmonic mappings, and in particular for et $\left(\mathbb{C}^{2}\right)$.

We recall that Definition 16 required also two additional topological properties; namely, the open set $D$ should satisfy $\operatorname{int}(\bar{D})=D ; \bar{D}$ is compact (all in the strong topology). These automatically exclude the set $E$ that was constructed in Definition 24. However, we can modify this construction to get at least an open set.

Proposition 27. Let $\Gamma$ be any family of holomorphic local homeomorphisms $F: \mathbb{C}^{2} \rightarrow \mathbb{C}^{2}$. Let $U$ be any open subset of 2 with a smooth boundary that contains the compact $E$. Then the open set $U-E$ is a characteristic set of $\Gamma$.
Proof. Since $E$ can not be mapped in the smooth $\partial U$ by an holomorphic local homeomorphism, we have for any $F_{1}, F_{2} \in$ $\Gamma$ for which $F_{1}(U-E)=F_{2}(U-E)$ that also $F_{1}(E)=F_{2}(E)$. Now the result follows by Proposition 25.

Remark 28. We note that if $\bar{U}$ is a compact then $U-E$ satisfies, at least, the requirement that $\overline{U-E}$ is compact. However, the "no-slit" condition $\operatorname{int}(\overline{U-E})=\operatorname{int}(\bar{U}) \neq U-E$ fails.

Now that we gained some experience with the topological construction of $E$ we are going to make one more step and fix its shortcomings that were mentioned above. We need to construct a domain $D$ of $\mathbb{C}^{2}$ which has the following three properties.

(1) $\operatorname{int}(\bar{D})=D$ relative to the complex topology.

(2) $\bar{D}$ is a compact subset of $\mathbb{C}^{2}$ relative to the strong topology.

(3) $\forall G_{1}, G_{2} \in \operatorname{et}\left(\mathbb{C}^{2}\right), G_{1}(D)=G_{2}(D) \Leftrightarrow G_{1} \equiv G_{2}$.

The complex topology and the strong topology are the same. Our construction will be a modification of the construction of the domain that was constructed in Proposition 27. We start by modifying the notion of an $m$-star that was introduced in Definition 23 .

Definition 29. Let $m$ be a natural number and $\alpha \in \mathbb{C}^{n}$. A thick $m$-star at $\alpha$ is a union of $2 m$ triangles, so that any pair intersects exactly at one vertex, and this vertex (i.e., common to all the $2 m$ triangles) is $\alpha$.

Definition 30. Let $E$ be the construction of Definition 24 that uses thick $m$-stars.

Proposition 31. Let $\Gamma$ be any family of holomorphic local homeomorphisms $F: \mathbb{C}^{2} \rightarrow \mathbb{C}^{2}$. Then $E$ is a characteristic set of $\Gamma$.

Proof. The proof is the same word by word as that of Proposition 25 where we replace $k$-star $S_{k}$ by thick $k$-star $S_{k}$.

We finally obtain our construction.

Proposition 32. Let $\Gamma$ be any family of holomorphic local homeomorphisms $F: \mathbb{C}^{2} \rightarrow \mathbb{C}^{2}$. Let $B(0, R)$ be an open ball centered at 0 with a radius $R$ large enough so that $E \subset B(0, R)$ (where $E$ is the set in Proposition 31). Then the domain $D=$ $B(0, R)-E$ is a characteristic set of $\Gamma$.

Proof. The proof is the same as that of Proposition 27 where we replace $k$-star $S_{k}$ by thick $k$-star $S_{k}$.

\section{Injectivity of the Left Composition Operator $L_{F}$}

We would like our natural mappings, the right mapping $R_{F}$ and the left mapping $L_{F}$, to be say bi-Lipschitz with respect to the metric $\rho_{D}$ (that reflects the fact that our mappings et $\left(\mathbb{C}^{2}\right)$ 
satisfy the Jacobian condition). Considering first the right mapping $R_{F}$, it would mean that given three étale mappings $G_{1}, G_{2}, F \in$ et $\left(\mathbb{C}^{2}\right)$ and a characteristic set $D$ of et $\left(\mathbb{C}^{2}\right)$ we need to compare the volume of $G_{1}(D) \Delta G_{2}(D)$ (multiplicities of $G_{1}$ and of $G_{2}$ are taken into account) with the volume of the $R_{F}$ deformed set $\left(G_{1} \circ F\right)(D) \Delta\left(G_{2} \circ F\right)(D)$. A short reflection shows that the two volumes are not comparable (in the sense of bi-Lipschitz). The situation is completely different when we replace the right mapping, $R_{F}$ by the left mapping, $L_{F}$. For example, we have the following.

Proposition 33. $\forall F \in$ Aut $\left(\mathbb{C}^{2}\right)$ the mapping $L_{F}$ is an isometry of the metric space ( et $\left.\left(\mathbb{C}^{2}\right), \rho_{D}\right)$.

Proof. For any two mappings $G_{1}$ and $G_{2}$ in et $\left(\mathbb{C}^{2}\right)$ we need to compare $\rho_{D}\left(G_{1}, G_{2}\right)$ with $\rho_{D}\left(F \circ G_{1}, F \circ G_{2}\right)$. We have (using our assumption on $F$ )

$$
\left(F \circ G_{1}\right)(D) \Delta\left(F \circ G_{2}\right)(D)=F\left(G_{1}(D) \Delta G_{2}(D)\right) .
$$

Since $F$ is also (globally) volume preserving we have

$$
\begin{aligned}
& \text { the volume of } F\left(G_{1}(D) \Delta G_{2}(D)\right) \\
& =\text { the volume of }\left(G_{1}(D) \Delta G_{2}(D)\right) .
\end{aligned}
$$

This proves that $\rho_{D}\left(G_{1}, G_{2}\right)=\rho_{D}\left(F \circ G_{1}, F \circ G_{2}\right)$.

We now drop the restrictive assumption that $F \in$ $\operatorname{Aut}\left(\mathbb{C}^{2}\right)$. Thus we merely have $F \in \operatorname{et}\left(\mathbb{C}^{2}\right)$ and we still want to compare $\rho_{D}\left(G_{1}, G_{2}\right)$ with $\rho_{D}\left(F \circ G_{1}, F \circ G_{2}\right)$, for any pair $G_{1}, G_{2} \in \operatorname{et}\left(\mathbb{C}^{2}\right)$. We only know that $F$ is a local diffeomorphism of $\mathbb{C}^{2}$ and (by the Jacobian condition) that it preserves (locally) the volume. In this case the geometrical degree of $F, d_{F}$ can be larger than 1 . We have the identity $d_{F}=\left|F^{-1}(\{(a, b)\})\right|$ which holds generically (in the Zariski sense) in $(a, b) \in \mathbb{C}^{2}$. Hence the (complex) dimension of the set $\left\{(a, b) \in \mathbb{C}^{2}|| F^{-1}(a, b) \mid<d_{F}\right\}$ is at most 1 . The Jacobian condition $\operatorname{det} J_{F} \equiv 1$ implies (as we noticed before) that $F$ preserves volume taking into account the multiplicity. The multiplicity is a result of the possibility that $F$ is not injective and hence the deformation of the characteristic set $D$ by $F$ convolves (i.e., might overlap at certain locations). However, this overlapping is bounded above by $d_{F}$. So if $A \subseteq \mathbb{C}^{2}$ is a measurable subset of $\mathbb{C}^{2}$ and we compare the volume of $A$ with the volume of its image $F(A)$, then

the volume of $F(A) \leq$ the volume of $A$

$$
\leq d_{F} \cdot\{\text { the volume of } F(A)\} .
$$

This can be rewritten as follows:

$$
\frac{1}{d_{F}} \cdot\{\text { the volume of } A\} \leq \text { the volume of } F(A)
$$

$\leq$ the volume of $A$.

This is the place to emphasize also the following conclusion (that follows by the generic identity $d_{F}=\left|F^{-1}(\{(a, b)\})\right|$ ); namely,

$$
\lim _{A \rightarrow \mathbb{C}^{2}} \frac{\text { the volume of } F(A)}{\text { the volume of } A}=\frac{1}{d_{F}},
$$

provided that the set $A$ tends to cover the whole of the complex space $\mathbb{C}^{2}$ in an appropriate manner. To better understand why the quotient tends to the lower limit $1 / d_{F}$ rather than to any number in the interval $\left[1 / d_{F}, 1\right]$ (if at all) we recall that our mapping belongs to et $\left(\mathbb{C}^{2}\right)$ and so is a polynomial étale mapping. So any point $(a, b) \in \mathbb{C}^{2}$ for which $\left|F^{-1}(a, b)\right|<d_{F}$ is an asymptotic value of $F$ and hence belongs to the curve $A_{F}$ which is the asymptotic variety of $F$. In other words the identity $d_{F}=\left|F^{-1}(a, b)\right|$ is satisfied exactly on the semialgebraic set $\mathbb{C}^{2}-A_{F}$ which is the complement of an algebraic curve. We now state and prove the main result of this paper.

Theorem 34. Let $F, G_{1}, G_{2} \in$ et $\left(\mathbb{C}^{2}\right)$. Then one has the following.

(i) $\rho_{D}\left(F \circ G_{1}, F \circ G_{2}\right) \leq \rho_{D}\left(G_{1}, G_{2}\right)$.

(ii) Suppose that $D$ is a family of characteristic sets of et $\left(\mathbb{C}^{2}\right)$ such that $D \rightarrow \mathbb{C}^{2}$, then $\forall \epsilon>0$ one has

$$
\left(\frac{1}{d_{F}}-\epsilon\right) \cdot \rho_{D}\left(G_{1}, G_{2}\right) \leq \rho_{D}\left(F \circ G_{1}, F \circ G_{2}\right)
$$

for $D$ being large enough.

(iii) Under the assumptions in (ii) one has

$$
\lim _{D \rightarrow \mathbb{C}^{2}} \frac{\rho_{D}\left(F \circ G_{1}, F \circ G_{2}\right)}{\rho_{D}\left(G_{1}, G_{2}\right)}=\frac{1}{d_{F}} .
$$

In particular, the left mapping $L_{F}:$ et $\left(\mathbb{C}^{2}\right) \rightarrow$ et $\left(\mathbb{C}^{2}\right)$, $L_{F}(G)=F \circ G$, is a bi-Lipschitz self-mapping of the metric space ( et $\left.\left(\mathbb{C}^{2}\right), \rho_{D}\right)$ with the constants $1 / d_{F} \leq 1$.

Proof. (i) $x \in\left(F \circ G_{1}\right)(D) \Delta\left(F \circ G_{2}\right)(D) \Rightarrow \exists y \in G_{j}(D)$, $j=1$ or 2 such that $x=F(y)$ and $x \notin\left(F \circ G_{3-j}\right)(D)$. By $x \notin\left(F \circ G_{3-j}\right)(D)$ it follows that $y \notin G_{3-j}(D)$ and so $y \in G_{1}(D) \Delta G_{2}(D)$ and $x=F(y) \in F\left(G_{1}(D) \Delta G_{2}(D)\right)$. Hence $\left(F \circ G_{1}\right)(D) \Delta\left(F \circ G_{2}\right)(D) \subseteq F\left(G_{1}(D) \Delta G_{2}(D)\right)$, so $\operatorname{vol}\left(\left(F \circ G_{1}\right)(D) \Delta\left(F \circ G_{2}\right)(D)\right) \leq \operatorname{vol}\left(F\left(G_{1}(D) \Delta G_{2}(D)\right)\right.$, and finally $\rho_{D}\left(F \circ G_{1}, F \circ G_{2}\right) \leq \rho_{D}\left(G_{1}, G_{2}\right)$.

(ii) and (iii). Here the proof is not just set theoretic. We will elaborate more in the remark that follows this proof. We recall that $F, G_{1}, G_{2} \in \operatorname{et}\left(\mathbb{C}^{2}\right)$. This implies that $\forall(\alpha, \beta) \in \mathbb{C}^{2}$ we have $\left|F^{-1}(\alpha, \beta)\right| \leq[\mathbb{C}(X, Y): \mathbb{C}(F)]$, the extension degree of $F$; see [3]. This is the so-called Fiber theorem for étale mappings. Moreover the image is cofinite; $\left|\mathbb{C}^{2}-F\left(\mathbb{C}^{2}\right)\right|<\infty$ [3]. Also $F$ has a finite set of exactly $d_{F}$ maximal domains $\left\{\Omega_{1}, \ldots, \Omega_{d_{F}}\right\}$. This means that $F$ is injective on each maximal domain $\Omega_{j}$, and $i \neq j \Rightarrow \Omega_{i} \cap \Omega_{j}=\emptyset$, and $\mathbb{C}^{2}=$ $\bigcup_{j=1}^{d_{F}} \overline{\Omega_{j}}$ and the boundaries $\partial \Omega_{j}$ are piecewise smooth (even piecewise analytic). For the theory of maximal domains of entire functions in one complex variable see [9], and for that theory for meromorphic functions in one complex variable see $[10,11]$. Here we use only basic facts of the theory which are valid also for more than complex variable. If $D$ is a family of characteristic sets of et $\left(\mathbb{C}^{2}\right)$ such that $D \rightarrow \mathbb{C}^{2}$, then by the 
above $G_{1}(D), G_{2}(D) \rightarrow \mathbb{C}^{2}-A$, where $A$ is a finite set, and if $G_{1} \not \equiv G_{2}$, then we have the identity

$$
\begin{gathered}
F\left(G_{1}(D) \Delta G_{2}(D)\right)-\left(F \circ G_{1}\right)(D) \Delta\left(F \circ G_{2}(D)\right) \\
=\left\{x=F(y)=F(z) \mid y \in G_{1}(D)-G_{2}(D)\right. \\
\left.\wedge z \in G_{2}(D)-G_{1}(D)\right\} .
\end{gathered}
$$

Recalling that $\left.\left(F \circ G_{1}\right)(D) \Delta\left(F \circ G_{2}(D)\right) \subseteq F\left(G_{1}(D) \Delta G_{2}(D)\right)\right)$ we write the last identity as follows:

$$
\begin{aligned}
F( & \left.G_{1}(D) \Delta G_{2}(D)\right) \\
= & \left(F \circ G_{1}\right)(D) \Delta\left(F \circ G_{2}(D)\right) \\
\cup & \left\{x=F(y)=F(z) \mid y \in G_{1}(D)-G_{2}(D)\right. \\
& \left.\wedge z \in G_{2}(D)-G_{1}(D)\right\} .
\end{aligned}
$$

Taking any two points $y \in G_{1}(D)-G_{2}(D)$ and $z \in G_{2}(D)-$ $G_{1}(D)$ (as in the defining equation of the set on the righthand side in the last identity), we note that there are $i \neq j$, $1 \leq i, j \leq d_{F}$ such that $y \in \Omega_{i} \wedge z \in \Omega_{j}$ (for $\left.F(y)=F(z) !\right)$. For $\widetilde{D}$ a large enough characteristic set of et $\left(\mathbb{C}^{2}\right)$, we will have $z \in G_{1}(\widetilde{D})$ and $y \in G_{2}(\widetilde{D})$ and so $y, z \in G_{1}(\widetilde{D}) \cap$ $G_{2}(\widetilde{D})$ (since $G_{1}(D), G_{2}(D) \rightarrow \mathbb{C}^{2}-\{$ a finite set $\}$ ). Hence $F\left(G_{1}(\widetilde{D}) \Delta G_{2}(\widetilde{D})\right)-\left(F \circ G_{1}\right)(\widetilde{D}) \Delta\left(F \circ G_{2}\right)(\widetilde{D})$ will not include the point $x$. We conclude that if $y$ and $z$ are $F$-equivalent $(F(y)=F(z))$, then $x=F(y)=F(z)$ will not belong to $F\left(G_{1}(D) \Delta G_{2}(D)\right)-\left(F \circ G_{1}\right)(D) \Delta\left(F \circ G_{2}\right)(D)$ for large enough $D$. We obtain the following crude estimate:

$$
\begin{aligned}
\operatorname{vol}( & \left\{x=F(y)=F(z) \mid y \in G_{1}(D)-G_{2}(D)\right. \\
& \left.\left.\wedge z \in G_{2}(D)-G_{1}(D)\right\}\right) \\
= & o\left(\operatorname{vol}\left(\left(F \circ G_{1}\right)(D) \Delta\left(F \circ G_{2}(D)\right)\right)\right) .
\end{aligned}
$$

One can think of $D$ as a large open ball centered at the origin of $\mathbb{R}^{4}, D \approx B(R)$ and with the radius $R$ and look at the images of the two polynomial étale mappings $\left(F \circ G_{1}\right)(B(R))$ and $(F \circ$ $\left.G_{2}\right)(B(R))$ and compare the volume of $\left(F \circ G_{1}\right)(B(R)) \Delta(F \circ$ $\left.G_{2}\right)(B(R))$ which is of the order of magnitude $R^{4 d}$, where $d$ depends on the algebraic degrees of $F \circ G_{1}$ and $F \circ G_{2}$, with the volume of the set in the left-hand side of the last equation. Similar estimates are used in the theory of covering surfaces by Ahlfors, see [12, chapter 5]. We conclude that

$$
\lim _{D \rightarrow \mathbb{C}^{2}} \frac{\operatorname{vol}\left(F\left(G_{1}(D) \Delta G_{2}(D)\right)\right)}{\operatorname{vol}\left(\left(F \circ G_{1}\right)(D) \Delta\left(F \circ G_{2}\right)(D)\right)}=1 .
$$

Hence

$$
\begin{aligned}
\lim _{D \rightarrow \mathbb{C}^{2}} \frac{\rho_{D}\left(F \circ G_{1}, F \circ G_{2}\right)}{\rho_{D}\left(G_{1}, G_{2}\right)} \\
=\lim _{D \rightarrow \mathbb{C}^{2}} \frac{\operatorname{vol}\left(\left(F \circ G_{1}\right)(D) \Delta\left(F \circ G_{2}\right)(D)\right)}{\operatorname{vol}\left(G_{1}(D) \Delta G_{2}(D)\right)} \\
=\lim _{D \rightarrow \mathbb{C}^{2}} \frac{\operatorname{vol}\left(\left(F \circ G_{1}\right)(D) \Delta\left(F \circ G_{2}\right)(D)\right)}{\operatorname{vol}\left(F\left(G_{1}(D) \Delta G_{2}(D)\right)\right)} \\
\quad \cdot \frac{\operatorname{vol}\left(F\left(G_{1}(D) \Delta G_{2}(D)\right)\right)}{\operatorname{vol}\left(G_{1}(D) \Delta G_{2}(D)\right)} \\
=1 \cdot \frac{1}{d_{F}}=\frac{1}{d_{F}} .
\end{aligned}
$$

Remark 35. The facts we used in proving (ii) and (iii) for étale mappings are in fact true in any dimension $n$, that is, in $\mathbb{C}^{n}$. In dimension $n=2$ it turns out that the codimension of the image of the mapping is 0 and in fact the coimage is a finite set. Also the fibers are finite and have a uniform bound on their cardinality (one can get a less tight uniform bound by the Bezout theorem). Here are few well-known facts (which one can find in Hartshorne's book on Algebraic Geometry, $[13])$.

(1) The following two conditions are equivalent.

(a) The Jacobian condition: the determinant $\operatorname{det} J_{F}$ is a nonzero constant.

(b) The map $F^{*}$ is étale (in standard sense of algebraic geometry). In particular it is flat.

Let $F^{*}: Y \rightarrow X$ be étale. Let $X^{i m}:=F^{*}(Y) \subseteq X$.

(2) For every prime ideal $\wp \subseteq A(X=\operatorname{spec}(A))$, with residue field $k(\wp)$ the ring $B \otimes_{A} k(\wp)$ is finite over $k(\wp)$ $(Y=\operatorname{spec}(B))$.

(3) $F^{*}$ is a quasifinite mapping.

(4) The set $X^{i m}$ is open in $X$.

(5) For every point $x \in X(\mathbb{C})$ the fiber $\left(F^{*}\right)^{-1}(x)$ is a finite subset of $Y(\mathbb{C})$.

(6) The ring homomorphism $A \rightarrow B$ is injective, and the induced field extension $K \rightarrow L$ is finite.

(7) There is a nonempty open subset $X^{\text {fin }} \subseteq X^{\text {im }}$ such that on letting $Y^{\mathrm{fin}}:=\left(F^{*}\right)^{-1}\left(X^{\mathrm{fin}}\right) \subseteq Y$, the map of schemes $\left.F^{*}\right|_{Y^{\text {fin }}}: Y^{\text {fin }} \rightarrow X^{\text {fin }}$ is finite. For any point $x \in X^{\text {fin }}(\mathbb{C})$ we have the equality $d_{x}=d_{F^{*}}$ the geometrical degree of $F^{*}$.

(8) The dimension of the set $Z:=X-X^{i m}$ is at most $n-2$.

(9) If $X^{i m}=X-Z$ is affine, then $Z=\emptyset$ and $X^{i m}=X$.

Let $X_{c l}$ be the topological space which is the set $X(\mathbb{C}) \cong$ $\mathbb{C}^{n}$ given the classical topology. Similarly for $Y_{c l}$, the map of 
schemes $F^{*}: Y \rightarrow X$ induces a map of topological spaces $F_{c l}: Y_{c l} \rightarrow X_{c l}\left(F_{c l}=\left.f^{*}\right|_{Y(\mathbb{C})}\right)$.

(10) The map $F_{c l}: Y_{c l} \rightarrow X_{c l}$ is a local homeomorphism. An immediate conclusion from Theorem 34 is the following, Corollary 36. $\forall F \in$ et $\left(\mathbb{C}^{2}\right)$ the left mapping $L_{F}$ : et $\left(\mathbb{C}^{2}\right)$ $\rightarrow L_{F}\left(\right.$ et $\left.\left(\mathbb{C}^{2}\right)\right), L_{F}(G)=F \circ G$ is an injective mapping.

\section{Extending the Notion of Geometrical Degree}

In this section we will outline the fact that some of the notions and results that are related to geometrical degree of an étale mapping originate, in fact, in the more basic topological spaces (no algebraic or holomorphic structure is needed). We will skip most of the proofs (that are elementary).

Definition 37. Let $X$ be a topological space. The semigroup of all the continuous mappings, $F: X \rightarrow X$, will be denoted by $C(X)$. Here, as usual, the binary operation is composition of mappings.

Proposition 38. (1) Let $X$ be a topological space, $F \in C(X)$, and $\overline{F(X)}=X$. Then $R_{F}: C(X) \rightarrow C(X)$ is injective.

(2) Let $X$ be a topological space, $F \in C(X)$, and $R_{F}$ : $C(X) \rightarrow C(X)$ is injective. Then for any $G, H \in C(X)$, the property $\left.G\right|_{F(X)}=\left.H\right|_{F(X)}$ implies that $G \equiv H$; that is, any $G \in C(X)$ is determined by its restriction $\left.G\right|_{F(X)}$.

(3) Let $X$ be a topological space that has the following property: for any closed $C \subseteq X$ and any point $x \in X-C$ there exist two continuous mappings $F, G: X \rightarrow X$ such that $\left.F\right|_{C}=\left.G\right|_{C}$, but $F(x) \neq G(x)$.

Let $F \in C(X)$ be such that $R_{F}: C(X) \rightarrow C(X)$ is injective. Then $\overline{F(X)}=X$.

Remark 39. Proposition 8 follows.

We are ready to discuss the notion of the geometrical degree, $d_{F}$, of appropriate mappings in $C(X)$.

Lemma 40. Let $X$ be a topological space, $F \in C(X)$ and $n \in$ $\mathbb{Z}^{+} \cup\{0\}$. If $F$ is open, then the set $B_{n}=\left\{x \in X|| F^{-1}(x) \mid \leq n\right\}$ is closed.

Corollary 41. Let $X$ be a topological space, $F \in C(X), F$ is open, and $n \in \mathbb{Z}^{+}$. Then one has the following.

(1) $\left\{x \in X|| F^{-1}(x) \mid \neq n\right\}=B_{n-1} \cup\left(X-B_{n}\right)$, the union of a closed set and an open set.

(2) $\left\{x \in X|| F^{-1}(x) \mid=n\right\}=B_{n}-B_{n-1}=B_{n} \cap\left(X-B_{n-1}\right)$, the intersection of a closed set and an open set.

(3) If $d_{F}=\max \left\{\left|F^{-1}(x)\right| \mid x \in X\right\}<\infty$ exists, then $\left\{x \in X|| F^{-1}(x) \mid \neq d_{F}\right\}$ is a closed set.

Remark 42. $B_{n} \subseteq B_{n+1}$.

Definition 43. Let $X$ be a topological space, $F \in C(X), F$ is open, and the maximum $d_{F}=\max \left\{\left|F^{-1}(x)\right| \mid x \in X\right\}<\infty$ exists. Then we call $d_{F}$ the geometrical degree of $F$.
Example 44. If $X=\mathbb{C}^{2}$ with the complex topology and $F \in$ et $\left(\mathbb{C}^{2}\right)$, then we know that $d_{F}$ exists. We also know that the set $A_{F}=\left\{x \in \mathbb{C}^{2}|| F^{-1}(x) \mid<d_{F}\right\}$ is a plane algebraic curve (possibly empty). Thus it is closed in $\mathbb{C}^{2}$. Moreover it is also small because $\operatorname{dim} A_{F}<2=\operatorname{dim} \mathbb{C}^{2}$.

We need one more property to hold for our mappings, namely, that the fiber size will generically be $d_{F}$, that is, that the set of all $x \in X$ for which $d_{F}=\left|F^{-1}(x)\right|$ will be a large set measured in the topology of $X$. This leads us to the following.

Definition 45. Let $X$ be a topological space. We will denote by $E(X)$ the set of all the mappings $F: X \rightarrow X$ that have the following properties.

(1) $F \in C(X)$.

(2) $F$ is open.

(3) The maximum $d_{F}=\max \left\{\left|F^{-1}(x)\right| \mid x \in X\right\}<\infty$ exists.

(4) $\overline{X-B_{d_{F}-1}}=X$.

Proposition 46. Let $X$ be an Hausdorff space. Then one has the following.

(1) $E(X)$ is a semigroup with an identity (where the binary operation is composition of mappings). In fact Aut $(X) \subseteq E(X)$.

(2) $\forall F, G \in E(X), d_{F \circ G}=d_{F} \cdot d_{G}$.

Proof. Checking that $E(X)$ is closed for composition: $F, G \in$ $C(X) \Rightarrow F \circ G \in C(X)$. Also $F, G$ open $\Rightarrow F \circ G$ is open. If $x \in X$, then $\left|F^{-1}(x)\right| \leq d_{F}$ and $\forall y \in F^{-1}(x)$; we have $\left|G^{-1}(y)\right| \leq d_{G}$. Since $(F \circ G)^{-1}(x)=G^{-1}\left(F^{-1}(x)\right)$, it follows that $d_{F \circ G}$ exists and, in fact, that $d_{F \circ G} \leq d_{F} \cdot d_{G}$. This gives the first three properties in the last definition. We need to check that $\overline{X-B_{d_{\mathrm{F} O}-1}}=X$. For that matter it will be convenient to denote $S_{F}=\left\{x \in X|| F^{-1}(x)<d_{F}\right\}$. Let $x \in X-S_{G}=$ $X-B_{d_{\mathrm{G}}-1}$. This set is open and dense in $X$. By our definitions $G^{-1}(x)=\left\{a_{1}, a_{2}, \ldots, a_{d_{G}}\right\}$, a finite set of exactly $d_{G}$ points. Since $X$ is Hausdorff we can find $d_{G}$ open neighborhoods $V_{a_{1}}, V_{a_{2}}, \ldots, V_{a_{d_{G}}}$ of $a_{1}, a_{2}, \ldots, a_{d_{G}}$, respectively which are pairwise disjoint. The images $G\left(V_{a_{1}}\right), G\left(V_{a_{2}}\right), \ldots, G\left(V_{a_{d_{G}}}\right)$ are open (since $G$ is open) neighborhoods of the point $x=$ $G\left(a_{1}\right)=G\left(a_{2}\right)=\cdots=G\left(a_{d_{g}}\right)$. Let us take the intersection

$$
V=\bigcap_{j=1}^{d_{G}} G\left(V_{a_{j}}\right) .
$$

Then $V$ is an open neighborhood of $x$ and $G^{-1}(V)$ consists of $d_{G}$ neighborhoods $V_{1}, V_{2}, \ldots, V_{d_{G}}$ of $a_{1}, a_{2}, \ldots, a_{d_{G}}$. We define $U_{j}=V_{j}-S_{G}, j=1,2, \ldots, d_{G}$. Then since $S_{G}$ is closed and $S_{G}^{c}$ is open and dense in $X$, the $U_{j}$ 's are open and dense subsets of the $V_{j}$ 's. Since $G$ is continuous and $G\left(V_{j}\right)=V$, it follows that $G\left(U_{j}\right)$ are open and dense in $V$. We note that each point $y \in U_{j}$ is such that $\left|F^{-1}(y)\right|=d_{F}$ because $S_{F}$ is disjoint of $U_{j}$. 
Thus the point $x \in X-S_{G}$ has a neighborhood $V$ and $d_{G}$ open and dense subsets $G\left(U_{j}\right)$ of $V$ such that each point

$$
x^{\prime} \in \bigcap_{j=1}^{d_{G}} G\left(U_{j}\right)
$$

(this set is still open and dense in $V$ ) is such that $\left|G^{-1}\left(x^{\prime}\right)\right|=$ $d_{G}$ and each $a \in G^{-1}\left(x^{\prime}\right)$ is such that $\left|F^{-1}(a)\right|=d_{F}$. Hence $\left|(F \circ G)^{-1}\left(x^{\prime}\right)\right|=d_{F} \cdot d_{G}$. This proves both that $\overline{X-B_{d_{F \circ G}-1}}=X$ and that $d_{F \circ G}=d_{F} \cdot d_{G}$.

Corollary 47. Let $X$ be Hausdorff and $\mathscr{F}$ a semigroup of mappings $F: X \rightarrow X$ which are continuous and open and suppose that there is an absolute constant $c \in \mathbb{Z}^{+}$such that $\forall F \in \mathscr{F}, d_{F} \leq c$. Then the sets $X-B_{d_{F}-1}, F \in \mathscr{F}$ cannot be dense in $X$, unless $\forall F \in \mathscr{F}, d_{F}=1$.

Corollary 48. Let $X$ be Hausdorff and $\mathscr{F}$ a family of mappings $F: X \rightarrow X$ which are continuous and open and satisfy $\overline{X-B_{d_{\mathrm{F} \circ}-1}}=X, \forall F \in \mathscr{F}$ (in particular $\forall F \in \mathscr{F}, d_{F}<\infty$ ). Let $S=\langle\mathscr{F}\rangle$ be the semigroup generated by $\mathscr{F}$ (composition of mappings is the binary operation). Then $\forall F, G \in S, d_{F \circ G}=$ $d_{F} \cdot d_{G}$.

Definition 49. Let $X$ be an Hausdorff space. A mapping $F \in$ $E(X)$ is called a composite mapping if $\exists G, H \in E(X)-\operatorname{Aut}(X)$ such that $F=G \circ H$. A mapping $A \in E(X)-\operatorname{Aut}(X)$ is a prime mapping if it is not composite. This is equivalent to the following: if $A=B \circ C$ for some $B, C \in E(X)$, then $B \in \operatorname{Aut}(X) \vee C \in \operatorname{Aut}(X)$. The subset of $E(X)$ of all the prime mappings will be denoted by $E_{p}(X)$. Thus the set of all the composite mappings is $E(X)-E_{p}(X)$.

Proposition 50. $\forall F \in E(X)-E_{p}(X), d_{F}$ is not a prime integer. Equivalently, $\forall F \in E(X), d_{F}$ is a prime integer $\Rightarrow F \in E_{p}(X)$.

Theorem 51. (1) If $E(X)-$ Aut $(X) \neq \emptyset$, then $E_{p}(X) \neq \emptyset$.

(2) $\forall F \in E(X), \exists k \in \mathbb{Z}^{+} \cup\{0\}, \exists A_{0} \in$ Aut $(X), \exists P_{1}, \ldots$, $P_{k} \in E_{p}(X)$ such that $F=A_{0} \circ P_{1} \circ \cdots \circ P_{k}$.

Maybe few elementary examples are in place.

Example 52. In Definition 45 we take $X=\mathbb{C}$ with the complex topology and (with an abuse of notation) take $\mathbb{C}[T]-$ $\mathbb{C}$ for $E(X)$. Then Proposition 46(2), $d_{F \circ G}=d_{F} \cdot d_{G}$, is the elementary fact from algebra that $\forall P, Q \in \mathbb{C}[T]$ we have $\operatorname{deg} P \circ Q=\operatorname{deg} P \cdot \operatorname{deg} Q$.

A second example is given in Section 4 of this paper.

Example 53. In Definition 45 we take $X=\mathbb{C}^{2}$ with the complex topology, and $E(X)=\operatorname{et}\left(\mathbb{C}^{2}\right)$. Then the theory that was outlined in Propositions 12 and 14 and Theorem 15 is a special case of the above more general topological theory.

\section{Conflict of Interests}

The author declares that there is no conflict of interests regarding the publication of this paper.

\section{References}

[1] R. Peretz, "On the structure of the semigroup of entire étale mappings," Complex Analysis and Operator Theory, vol. 7, no. 5, pp. 1655-1674, 2013.

[2] H. Bass, E. H. Connell, and D. Wright, "The Jacobian conjecture: reduction of degree and formal expansion of the inverse," American Mathematical Society, Bulletin: New Series, vol. 7, no. 2, pp. 287-330, 1982.

[3] A. van den Essen, Polynomial Automorphisms and the Jacobian Conjecture, Progress in Mathematics, vol. 190, Birkhäuser, Berlin, Germany, 2000.

[4] S. Smale, "Mathematical problems for the next century," The Mathematical Intelligencer, vol. 20, no. 2, pp. 7-15, 1998.

[5] R. Peretz, "Picard theorems for Keller mappings in dimension two and the phantom curve," Journal of Mathematics Research, vol. 6, no. 1, 2014.

[6] S. Bochner and W. Martin, Several Complex Variables, Princeton University Press, Princeton, NJ, USA, 1948.

[7] T. Nishino, Function Theory in Several Complex Variables, vol. 193 of Translations of Mathematical Monographs, American Mathematical Society, Providence, RI, USA, 1996.

[8] T. W. Gamelin, Complex Analysis, Springer, New York, NY, USA, 2001.

[9] R. Peretz, "Maximal domains for entire functions," Journal d'Analyse Mathématique, vol. 61, pp. 1-28, 1993.

[10] T. Shimizu, "On the fundamental domains and the groups for meromorphic functions I," Japanese Journal of Mathematics, vol. 8, pp. 175-236, 1931.

[11] T. Shimizu, "On the fundamental domains and the groups for meromorphic functions II," Japanese Journal of Mathematics, vol. 8, pp. 237-309, 1931.

[12] W. K. Hayman, Meromorphic Functions, Oxford Mathematical Monographs, Clarendon Press, Oxford, UK, 1964.

[13] R. Hartshorne, Algebraic Geometry, vol. 52 of Graduate Texts in Mathematics, Springer, 1977. 


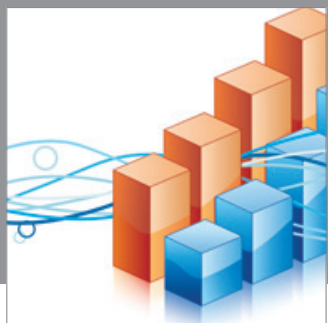

Advances in

Operations Research

mansans

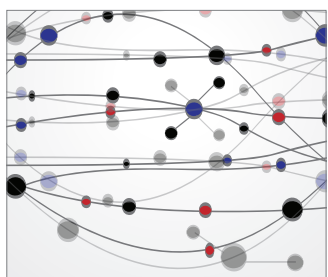

The Scientific World Journal
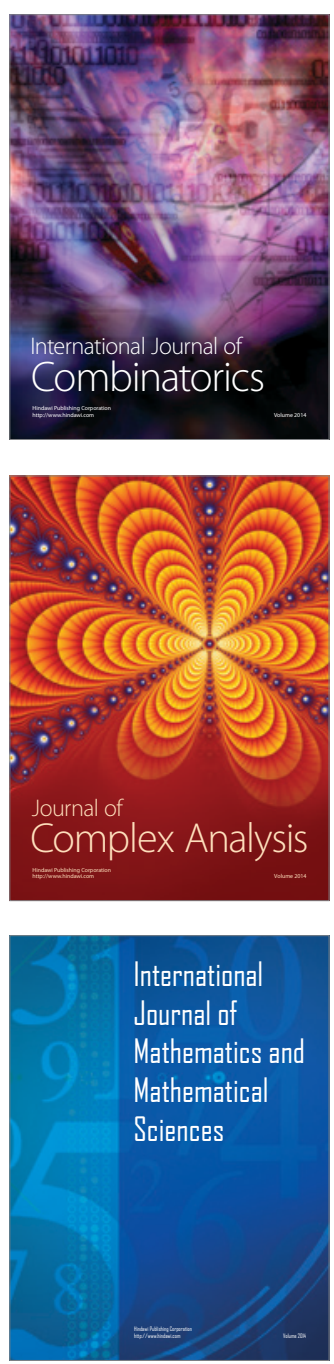
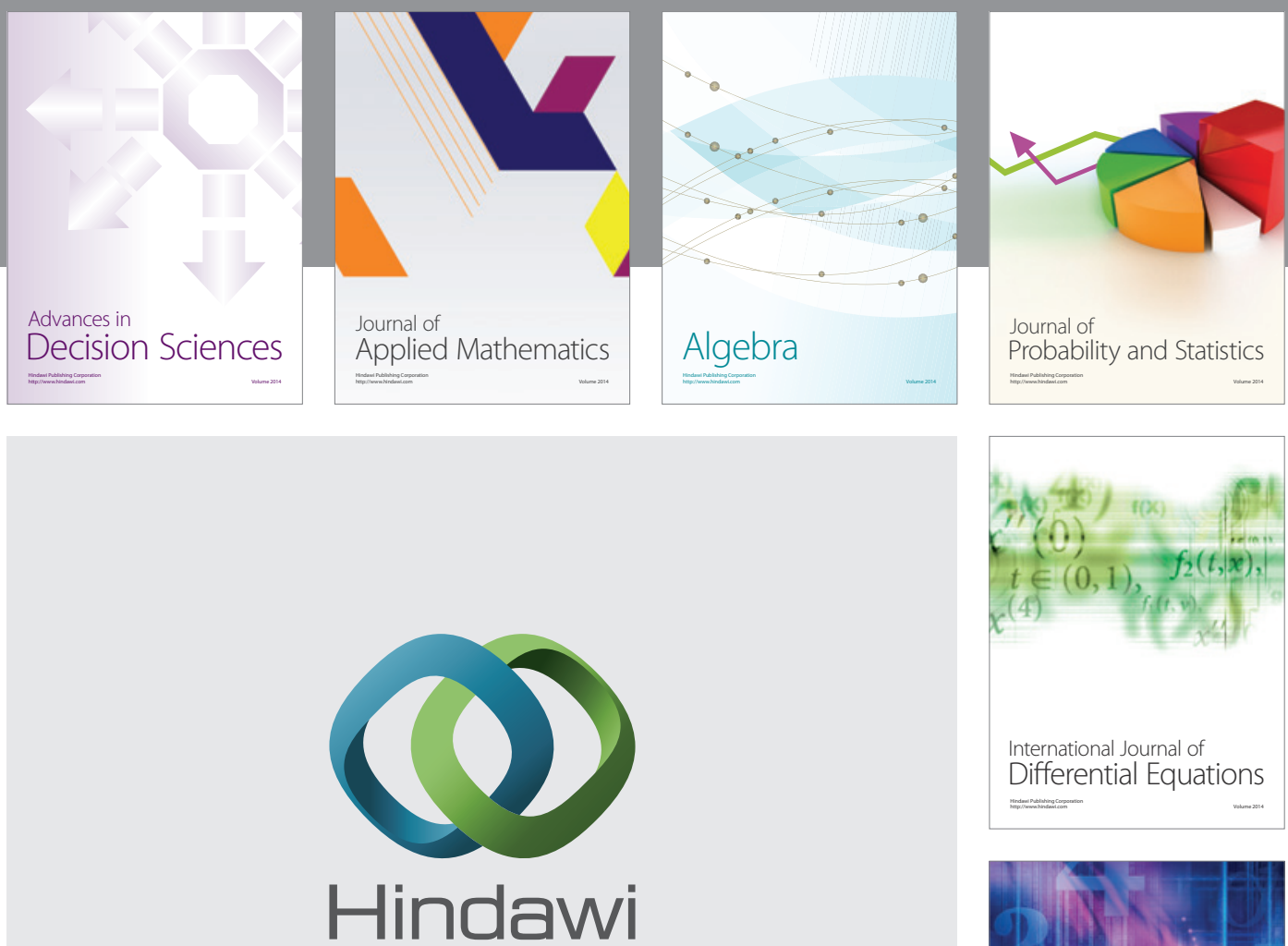

Submit your manuscripts at http://www.hindawi.com
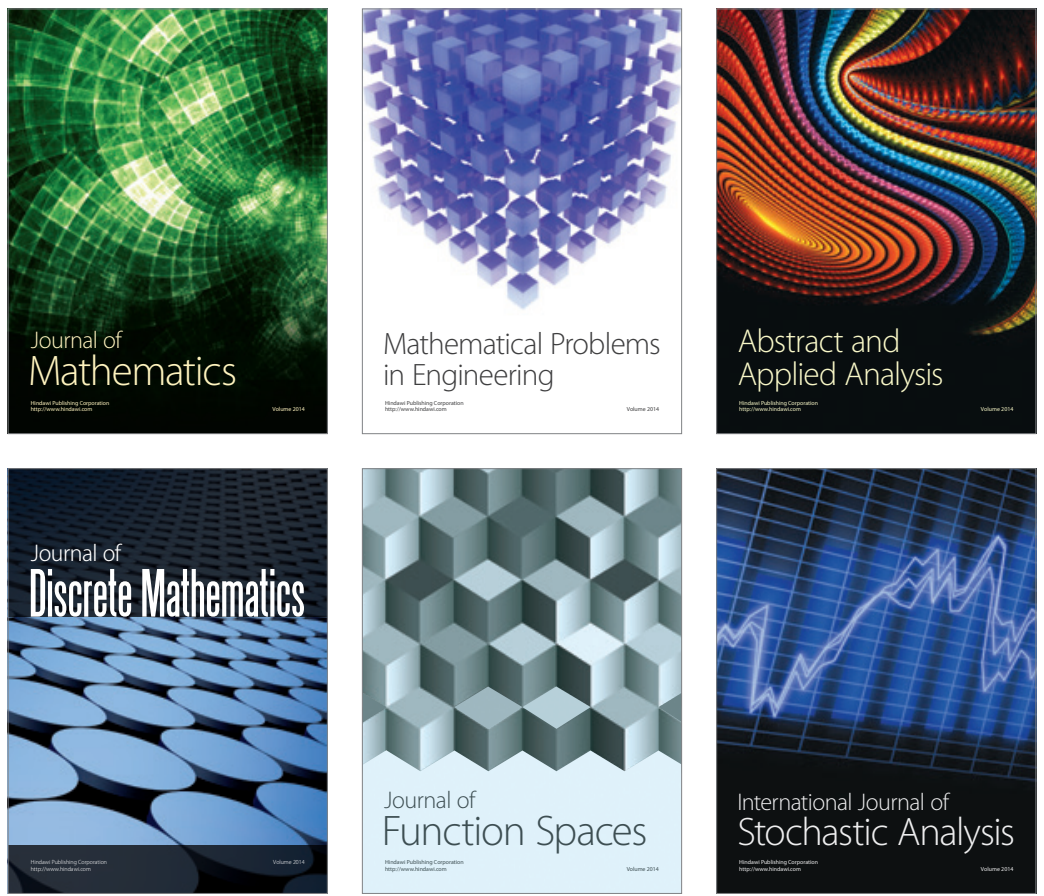

Journal of

Function Spaces

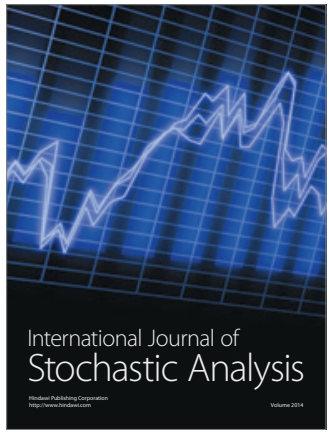

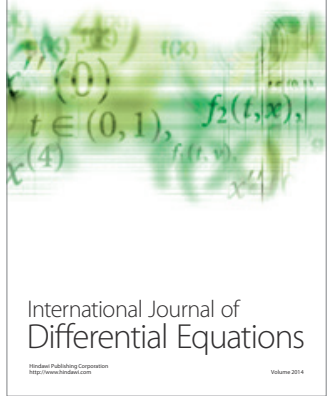
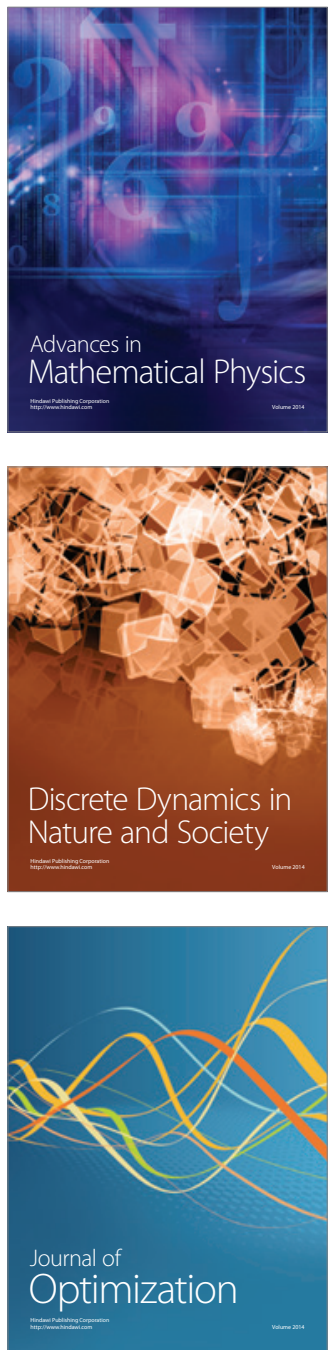